\title{
Algebra $g l(\lambda)$ Inside the Algebra of Differential Operators on the Real Line
}

\author{
H GARGOUBI \\ I.P.E.I.M., route de Kairouan, 5019 Monastir, Tunisia \\ E-mail: hichem.gargoubi@ipeim.rnu.tn \\ Received November 22, 2001; Revised February 26, 2002; Accepted March 12, 2002
}

\begin{abstract}
The Lie algebra $\operatorname{gl}(\lambda)$ with $\lambda \in \mathbb{C}$, introduced by B L Feigin, can be embedded into the Lie algebra of differential operators on the real line (see [7]). We give an explicit formula of the embedding of $\operatorname{gl}(\lambda)$ into the algebra $\mathcal{D}_{\lambda}$ of differential operators on the space of tensor densities of degree $\lambda$ on $\mathbb{R}$. Our main tool is the notion of projectively equivariant symbol of a differential operator.
\end{abstract}

\section{Introduction}

The Lie algebra $\operatorname{gl}(\lambda)(\lambda \in \mathbb{C})$ was introduced by B L Feigin in [7] for calculation the cohomology of the Lie algebra of differential operators on the real line. The algebra $\operatorname{gl}(\lambda)$ is defined as the quotient of the universal enveloping algebra $\mathrm{U}\left(\mathrm{sl}_{2}\right)$ of $\mathrm{sl}_{2}$ with respect to the ideal generated by the element $\Delta-\lambda(\lambda-1)$, where $\Delta$ is the Casimir element of $\mathrm{U}\left(\mathrm{sl}_{2}\right)$. $\operatorname{gl}(\lambda)$ is turned into a Lie algebra by the standard method of setting $[a, b]=a b-b a$.

According to Feigin, $\operatorname{gl}(\lambda)$ can be considered as an analogue of $\operatorname{gl}(n)$ for $n=\lambda \in \mathbb{N}$; it is also called the algebra of matrices of complex size, see also [13, 16, 17, 12].

We consider the space $\mathcal{D}_{\lambda}$ of all linear differential operators acting on tensor densities of degree $\lambda$ on $\mathbb{R}$. One of the main results of $[7$ is the construction of an embedding $\operatorname{gl}(\lambda) \rightarrow \mathcal{D}_{\lambda}$.

The purpose of this paper is to give an explicit formula of this embedding. We also show that this embedding realizes the isomorphism of Lie algebras $\operatorname{gl}(\lambda) \cong \mathcal{D}_{\lambda}^{\text {pol }}$ constructed in 1, 2], where $\mathcal{D}_{\lambda}^{\text {pol }} \subset \mathcal{D}_{\lambda}$ is the subalgebra of differential operators with polynomial coefficients.

The main idea of this paper is to use the projectively equivariant symbol of a differential operator, that is an $\mathrm{sl}_{2}$-equivariant way to associate a polynomial function on $T^{*} \mathbb{R}$ to a differential operator. The notion of projectively equivariant symbol was defined in [4, 15] and used in [8, 9, 10] for study of modules of differential operators. 


\section{Basic definitions}

2.1 The Lie algebra $\operatorname{gl}(\boldsymbol{\lambda})$. Let $\operatorname{Vect}(\mathbb{R})$ be the Lie algebra of smooth vector fields on $\mathbb{R}$ with complex coefficients: $X=X(x) \partial$, where $X(x)$ is a smooth complex function of one real variable; $X(x) \in C^{\infty}(\mathbb{R}, \mathbb{C})$, and where $\partial=\frac{d}{d x}$. Consider the Lie algebra $\mathrm{sl}_{2} \subset \operatorname{Vect}(\mathbb{R})$ generated by the vector fields

$$
\left\{\partial, x \partial, x^{2} \partial\right\} \text {. }
$$

Denote $e_{i}:=x^{i} \partial, i=0,1,2$, the Casimir element

$$
\Delta:=e_{1}^{2}-\frac{1}{2}\left(e_{0} e_{2}+e_{2} e_{0}\right)
$$

generates the center of $\mathrm{U}\left(\mathrm{sl}_{2}\right)$. The quotient

$$
\operatorname{gl}(\lambda):=\mathrm{U}\left(\mathrm{sl}_{2}\right) /(\Delta-\lambda(\lambda-1)), \quad \lambda \in \mathbb{C}
$$

is naturally a Lie algebra containing $\mathrm{sl}_{2}$.

2.2 Modules of differential operators on $\mathbb{R}$. Denote $\mathcal{D}$ the Lie algebra of linear differential operators on $\mathbb{R}$ with complex coefficients:

$$
A=a_{n}(x) \partial^{n}+a_{n-1}(x) \partial^{n-1}+\cdots+a_{0}(x),
$$

with $a_{i}(x) \in C^{\infty}(\mathbb{R}, \mathbb{C})$.

For $\lambda \in \mathbb{C}$, Vect $(\mathbb{R})$ is embedded into the Lie algebra $\mathcal{D}$ by:

$$
X \mapsto L_{X}^{\lambda}:=X(x) \partial+\lambda X^{\prime}(x)
$$

Denote $\mathcal{D}_{\lambda}$ the $\operatorname{Vect}(\mathbb{R})$-module structure with respect to the adjoint action of $\operatorname{Vect}(\mathbb{R})$ on $\mathcal{D}$. The module $\mathcal{D}_{\lambda}$ has a natural filtration: $\mathcal{D}_{\lambda}^{0} \subset \mathcal{D}_{\lambda}^{1} \subset \cdots \subset \mathcal{D}_{\lambda}^{n} \subset \cdots$, where $\mathcal{D}_{\lambda}^{n}$ is the module of $n$-th order differential operators (2.2).

Geometrically speaking, differential operators are acting on tensor densities, namely: $A: \mathcal{F}_{\lambda} \rightarrow \mathcal{F}_{\lambda}$, where $\mathcal{F}_{\lambda}$ is the space of tensor densities of degree $\lambda$ on $\mathbb{R}$ (i.e., of sections of the line bundle $\left.\left(T^{*} \mathbb{R}\right)^{\otimes \lambda}, \lambda \in \mathbb{C}\right)$, that is: $\phi=\phi(x)(d x)^{\lambda}$, where $\phi(x) \in C^{\infty}(\mathbb{R}, \mathbb{C})$.

It is evident that $\mathcal{F}_{\lambda} \cong C^{\infty}(\mathbb{R}, \mathbb{C}$ ) as linear spaces (but not as modules) for any $\lambda$. We use this identification throughout this paper. The Lie algebra structures of differential operators acting on the space of tensor densities and on the space of functions are also identified (see [8]).

The Vect $(\mathbb{R})$-modules $\mathcal{D}_{\lambda}$ were considered by classics (see [3, 18]) and, recently, studied in a series of papers [5, 9, 8, 10, 14.

2.3 Principal symbol. Let $\operatorname{Pol}\left(T^{*} \mathbb{R}\right)$ be the space of functions on $T^{*} \mathbb{R}$ polynomial in the fibers. This space is usually considered as the space of symbols associated to the space of differential operators on $\mathbb{R}$.

Recall that the principal symbol of a differential operator is the linear map $\sigma: \mathcal{D} \rightarrow$ $\operatorname{Pol}\left(T^{*} \mathbb{R}\right)$ defined by:

$$
\sigma(A)=a_{n}(x) \xi^{n}
$$

where $A$ is a differential operator (2.2) and $\xi$ is the coordinate on the fiber. 
One can also speak about the principal symbol of an element of $\mathrm{U}\left(\mathrm{sl}_{2}\right)$. Indeed, $\mathrm{U}\left(\mathrm{sl}_{2}\right)$ is canonically identified with the symmetric algebra $S\left(\mathrm{sl}_{2}\right)$ as $\mathrm{sl}_{2}$-modules (see, e.g., 6, p.82]). Using the realization (2.1), the algebra $S\left(\mathrm{sl}_{2}\right)$ can be projected to $\operatorname{Pol}\left(T^{*} \mathbb{R}\right)$. Therefore, one can define in a natural way the principal symbol on $S\left(\mathrm{sl}_{2}\right)$.

Our goal is to construct an $\mathrm{sl}_{2}$-equivariant linear map $T_{\lambda}: \mathrm{U}\left(\mathrm{sl}_{2}\right) \rightarrow \mathcal{D}_{\lambda}$ which preserves the principal symbol, i.e., such that the following diagram commutes:

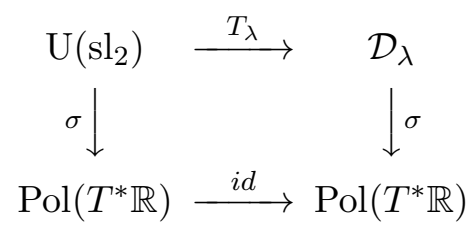

2.4 Projectively equivariant symbol. Viewed as a Vect $(\mathbb{R})$-module, the space of symbols corresponding to $\mathcal{D}_{\lambda}$ has the form:

$$
\operatorname{Pol}\left(T^{*} \mathbb{R}\right) \cong \mathcal{F}_{0} \oplus \mathcal{F}_{1} \oplus \cdots \oplus \mathcal{F}_{n} \oplus \cdots
$$

The space of polynomials of degree $\leq n$ is a submodule of $\operatorname{Pol}\left(T^{*} \mathbb{R}\right)$ which we denote $\operatorname{Pol}_{n}\left(T^{*} \mathbb{R}\right)$.

The following result of $\left[\underline{8}\right.$ allows one to identify, for arbitrary $\lambda, \mathcal{D}_{\lambda}^{n}$ with $\operatorname{Pol}_{n}\left(T^{*} \mathbb{R}\right)$ as $\mathrm{sl}_{2}$-modules:

(i) There exists a unique $\operatorname{sl}(2, \mathbb{R})$-isomorphism $\sigma_{\lambda}: \mathcal{D}_{\lambda}^{n} \rightarrow \operatorname{Pol}_{n}\left(T^{*} \mathbb{R}\right)$ preserving the principal symbol.

(ii) $\sigma_{\lambda}$ associates to each differential operator $A$ the polynomial $\sigma_{\lambda}(A)=\sum_{p=0}^{n} \bar{a}_{p}(x) \xi^{p}$, defined by:

$$
\bar{a}_{p}(x)=\sum_{j=p}^{n} \alpha_{p}^{j} a_{j}^{(j-p)},
$$

where the constants $\alpha_{p}^{j}$ are given by:

$$
\alpha_{p}^{j}=\frac{\left(\begin{array}{c}
j \\
p
\end{array}\right)\left(\begin{array}{c}
2 \lambda-p \\
j-p
\end{array}\right)}{\left(\begin{array}{c}
j+p+1 \\
2 p+1
\end{array}\right)}
$$

(the binomial coefficient $\left(\begin{array}{l}\lambda \\ j\end{array}\right)=\lambda(\lambda-1) \cdots(\lambda-j+1) / j$ ! is a polynomial in $\lambda$ ).

The isomorphism $\sigma_{\lambda}$ is called the projectively equivariant symbol map. Its explicit formula was first found in [4, 15] in the general case of pseudo-differential operators on a one-dimensional manifold (see also [15] for the multi-dimensional case).

\section{Main result}

In this section, we give the main result of this paper. We adopt the following notations:

$$
\left[L_{X_{1}}^{\lambda} L_{X_{2}}^{\lambda} \cdots L_{X_{n}}^{\lambda}\right]_{+}:=\sum_{\tau \in S_{n}} L_{X_{\tau(1)}}^{\lambda} \circ L_{X_{\tau(2)}}^{\lambda} \circ \cdots \circ L_{X_{\tau(n)}}^{\lambda}
$$


for a symmetric $n$-linear map from $\operatorname{Vect}(\mathbb{R})$ to $\mathcal{D}$ and

$$
\left(X_{1} X_{2} \cdots X_{n}\right)_{+}:=\sum_{\tau \in S_{n}} X_{\tau(1)} X_{\tau(2)} \cdots X_{\tau(n)}
$$

for a symmetric $n$-linear map from $\mathrm{sl}_{2}$ to $\mathrm{U}\left(\mathrm{sl}_{2}\right)$, where $S_{n}$ is the group of permutations of $n$ elements and $X_{i} \in \mathrm{sl}_{2}$.

Theorem 1. (i) For arbitrary $\lambda \in \mathbb{C}$, there exists a unique $\mathrm{sl}_{2}$-equivariant linear map preserving the principal symbol:

$$
T_{\lambda}: \mathrm{U}\left(\mathrm{sl}_{2}\right) \rightarrow \mathcal{D}_{\lambda}
$$

defined by

$$
T_{\lambda}\left(\left(X_{1} X_{2} \cdots X_{n}\right)_{+}\right)=\left[L_{X_{1}}^{\lambda} L_{X_{2}}^{\lambda} \cdots L_{X_{n}}^{\lambda}\right]_{+},
$$

where $X_{i} \in\left\{e_{0}, e_{1}, e_{2}\right\}, L_{X_{i}}^{\lambda}$ given by (2.3) and $n=1,2, \ldots$.

(ii) The operator $T_{\lambda}$ is given in term of the $\mathrm{sl}_{2}$-equivariant symbol (2.5) by:

$$
\sigma_{\lambda}\left(\left[L_{X_{1}}^{\lambda} L_{X_{2}}^{\lambda} \cdots L_{X_{n}}^{\lambda}\right]_{+}\right)=\sum_{\substack{0 \leq k \leq n \\ k \text { even }}} P_{k}^{n}(\lambda) \mathcal{A}_{k}\left(X_{1}, \ldots, X_{n}\right) \xi^{n-k},
$$

where

$$
\begin{aligned}
& \mathcal{A}_{k}\left(X_{1}, \ldots, X_{n}\right) \\
& \quad=\sum_{2 p+m=k}\left(\begin{array}{c}
k / 2 \\
p
\end{array}\right)(-2)^{p}\left(X_{1}^{\prime \prime} \ldots X_{p}^{\prime \prime} X_{p+1}^{\prime} \cdots X_{p+m}^{\prime} X_{p+m+1} \cdots X_{n}\right)_{+}
\end{aligned}
$$

and

$$
P_{k}^{n}(\lambda)=\sum_{p=0}^{n} \sum_{l=n-k}^{n}(l-n+k) ! \frac{\left(\begin{array}{c}
l \\
n-k
\end{array}\right)^{2}\left(\begin{array}{c}
2 \lambda-n+k \\
l-n+k
\end{array}\right)}{\left(\begin{array}{l}
n-k+l+1 \\
2 n-2 k+1
\end{array}\right)}\left(\begin{array}{l}
n \\
p
\end{array}\right)\left\{\begin{array}{l}
p \\
l
\end{array}\right\} \lambda^{n-p},
$$

where $\left\{\begin{array}{l}p \\ l\end{array}\right\}$ is the Stirling number of the second kind ${ }^{1}$.

It is worth noticing that the linear map $T_{\lambda}$ does not depend on the choice of the PBWbase in $\mathrm{U}\left(\mathrm{sl}_{2}\right)$.

\section{Proof of Theorem 1}

By construction, the linear map $T_{\lambda}$ is $\mathrm{sl}_{2}$-equivariant.

$4.1 \mathrm{sl}_{2}$-invariant symmetric differential operators. To prove part (ii) of Theorem 1 one needs the following

\footnotetext{
${ }^{1}$ We refer to 11$]$ as a nice elementary introduction to the combinatorics of the Stirling numbers.
} 
Proposition 1. For arbitrary $\mu \in \mathbb{C}$ and $n=1,2, \ldots$, there exists at most one, up to proportionality, $\mathrm{sl}_{2}$-equivariant symmetric operator $\otimes^{n} \mathrm{sl}_{2} \rightarrow \mathcal{F}_{\mu}$ which is differential with respect to the vector fields $X_{i} \in \mathrm{sl}_{2}$. This operator exists if and only if $\mu=k-n$, where $k$ is an even positive integer. It is denoted: $\mathcal{A}_{k}: \otimes^{n} \mathrm{sl}_{2} \rightarrow \mathcal{F}_{k-n}$, and defined by the expression (3.3).

Proof. Each $k$-th order differential operator $\mathcal{A}: \otimes^{n} \mathrm{sl}_{2} \rightarrow \mathcal{F}_{\mu}$ is of the form:

$$
\mathcal{A}\left(X_{1}, \ldots, X_{n}\right)=\sum_{2 p+m=k} \beta_{p}(x)\left(X_{1}^{\prime \prime} \cdots X_{p}^{\prime \prime} X_{p+1}^{\prime} \cdots X_{p+m}^{\prime} X_{p+m+1} \cdots X_{n}\right)_{+},
$$

where $\beta_{p}(x)$ are some functions.

The condition of $\mathrm{sl}_{2}$-equivariance for $\mathcal{A}$ reads as follows:

$$
X\left[\mathcal{A}\left(X_{1}, \ldots, X_{n}\right)\right]^{\prime}+\mu X^{\prime} \mathcal{A}\left(X_{1}, \ldots, X_{n}\right)=\sum_{i=1}^{n} \mathcal{A}\left(X_{1}, \ldots, L_{X}^{-1}\left(X_{i}\right), \ldots, X_{n}\right),
$$

where $X \in \mathrm{sl}_{2}$.

Substitute $X=\partial$ to check that the coefficients $\beta_{p}(x)$ do not depend on $x$. Substitute $X=x \partial$ to obtain the condition $\mu=k-n$. At last, substitute $X=x^{2} \partial$ and put $\beta_{0}=1$ to obtain, for even $k$, the coefficients from (3.3). If $k$ is odd, one obtains $\beta_{p}=0$ for all $p$.

Proposition 1 is proven.

The general form (3.2) is a consequence of Proposition 1 and decomposition (2.4).

4.2 Polynomials $\boldsymbol{P}_{\boldsymbol{k}}^{\boldsymbol{n}}(\boldsymbol{\lambda})$. To compute the polynomials $P_{k}^{n}$, put $X_{1}=\cdots=X_{n}=x \partial$. One readily gets, from (3.2),

$$
\left.\sigma_{\lambda}\left(T_{\lambda}\left(X_{1}, \ldots, X_{n}\right)\right)\right|_{x=1}=n ! \sum_{\substack{0 \leq k \leq n \\ k \text { even }}} P_{k}^{n}(\lambda) \xi^{n-k} .
$$

Furthermore, using the well-known expression $(x \partial)^{n}=\sum_{l=0}^{n}\left\{\begin{array}{l}n \\ l\end{array}\right\} x^{l} \partial^{l}$, one has:

$$
\begin{aligned}
T_{\lambda}\left(X_{1}, \ldots, X_{n}\right) & =n !(x \partial+\lambda)^{n} \\
& =n ! \sum_{p=0}^{n}\left(\begin{array}{l}
n \\
p
\end{array}\right)(x \partial)^{n} \lambda^{n-p}=n ! \sum_{p=0}^{n} \sum_{l=0}^{n}\left(\begin{array}{l}
n \\
p
\end{array}\right)\left\{\begin{array}{l}
n \\
l
\end{array}\right\} x^{l} \partial^{l} \lambda^{n-p} .
\end{aligned}
$$

A straightforward computation gives the projectively equivariant symbol (2.5) of this differential operator:

$$
\begin{aligned}
& \left.\sigma_{\lambda}\left(T_{\lambda}\left(X_{1}, \ldots, X_{n}\right)\right)\right|_{x=1} \\
& =n ! \sum_{\substack{0 \leq k \leq n \\
k \text { even }}} \sum_{p=0}^{n} \sum_{l=n-k}^{n}(l-n+k) ! \frac{\left(\begin{array}{c}
l \\
n-k
\end{array}\right)^{2}\left(\begin{array}{c}
2 \lambda-n+k \\
l-n+k
\end{array}\right)}{\left(\begin{array}{c}
n-k+l+1 \\
2 n-2 k+1
\end{array}\right)}\left(\begin{array}{l}
n \\
p
\end{array}\right)\left\{\begin{array}{l}
p \\
l
\end{array}\right\} \lambda^{n-p} \xi^{n-k} .
\end{aligned}
$$

Compare with the equality (4.1) to obtain the formulae from (3.4).

Theorem 1 (ii) is proven. 
4.3 Uniqueness. Let $T$ be an $\mathrm{sl}_{2}$-equivariant linear map $\mathrm{U}\left(\mathrm{sl}_{2}\right) \rightarrow \mathcal{D}_{\lambda}$ for a certain $\lambda \in \mathbb{C}$. In view of the decomposition (2.4), it follows from Proposition 1 that $\left.\sigma_{\lambda} \circ T\right|_{\mathcal{F}_{k}}=$ $c_{k}(\lambda) \mathcal{A}_{k}$, where $c_{k}(\lambda)$ is a constant depending on $\lambda$. Recall that $\operatorname{Pol}_{n}\left(T^{*} \mathbb{R}\right)$ is a rigid $\mathrm{sl}_{2^{-}}$ module, i.e., every $\mathrm{sl}_{2}$-equivariant linear map on $\operatorname{Pol}_{n}\left(T^{*} \mathbb{R}\right)$ is proportional to the identity (see, e.g., [15]). Assuming, now, that $T$ preserves the principal symbol, the rigidity of $\operatorname{Pol}_{n}\left(T^{*} \mathbb{R}\right)$ fixes the constants $c_{k}(\lambda)$ in a unique way. Hence the uniqueness of $T_{\lambda}$.

Theorem 1 is proven.

\section{$5 \quad$ The embedding $\mathrm{gl}(\lambda) \rightarrow \mathcal{D}_{\lambda}$}

A corollary of the uniqueness of the operator $T_{\lambda}$ and results of [1, 2, 1, 17] is that the embedding $\mathrm{gl}(\lambda) \rightarrow \mathcal{D}_{\lambda}$ constructed in [7] coincides with $T_{\lambda}$.

More precisely, according to results of [1, 2, 17, there exists a homomorphism of Lie algebras $p_{\lambda}: \mathrm{U}\left(\mathrm{sl}_{2}\right) \rightarrow \mathcal{D}_{\lambda}$ preserving the principal symbol. The homomorphism $p_{\lambda}$ is, in particular, $\mathrm{sl}_{2}$-equivariant. By uniqueness of $T_{\lambda}$, one has $T_{\lambda}=p_{\lambda}$. It is also proven that the kernel of $p_{\lambda}$ is a two-sided ideal of $\mathrm{U}\left(\mathrm{sl}_{2}\right)$ generated by $\Delta-\lambda(\lambda-1)$ (see [1, 2]). Taking the quotient, one then has an embedding $\tilde{T}_{\lambda}: \operatorname{gl}(\lambda) \rightarrow \mathcal{D}_{\lambda}$. Since the embedding from [7] preserves the principal symbol, it is equal to $\tilde{T}_{\lambda}$. Finally, it is obvious that the image of $T_{\lambda}$ is the subalgebra $\mathcal{D}_{\lambda}^{\text {pol }} \subset \mathcal{D}_{\lambda}$ of differential operators with polynomial coefficients. Therefore, $\tilde{T}_{\lambda}: \operatorname{gl}(\lambda) \rightarrow \mathcal{D}_{\lambda}^{\text {pol }}$ is a Lie algebras isomorphism.

\section{$6 \quad$ Examples}

As an illustration of Theorem 1 let us give the expressions of the general formulae (3.1) and (3.2) for the order $n=1,2,3,4,5$. Let $X_{1}, X_{2}, X_{3}, X_{4}$ and $X_{5}$ be arbitrary vector fields in $\mathrm{sl}_{2}$.

1) The $\mathrm{sl}_{2}$-equivariant symbol, defined by (2.5), of a first order operator of a Lie derivative $L_{X_{1}}^{\lambda}$ is

$$
\sigma_{\lambda}\left(L_{X_{1}}^{\lambda}\right)=X_{1}(x) \xi
$$

2) The "anti-commutator" $\left[L_{X_{1}}^{\lambda} L_{X_{2}}^{\lambda}\right]_{+}$has the following projectively equivariant symbol:

$$
\sigma_{\lambda}\left(\left[L_{X_{1}}^{\lambda} L_{X_{2}}^{\lambda}\right]_{+}\right)=\left(X_{1} X_{2}\right)_{+} \xi^{2}+\frac{1}{3} \lambda(\lambda-1)\left(\left(X_{1}^{\prime} X_{2}^{\prime}\right)_{+}-2\left(X_{1}^{\prime \prime} X_{2}\right)_{+}\right)
$$

which also following from (2.5).

3) The projectively equivariant symbol of a third order expression $\left[L_{X_{1}}^{\lambda} L_{X_{2}}^{\lambda} L_{X_{3}}^{\lambda}\right]_{+}$can be also easily calculated from (2.5). The result is:

$$
\begin{aligned}
\sigma_{\lambda}([ & \left.\left.L_{X_{1}}^{\lambda} L_{X_{2}}^{\lambda} L_{X_{3}}^{\lambda}\right]_{+}\right)=\left(X_{1} X_{2} X_{3}\right)_{+} \xi^{3} \\
& +\frac{1}{5}\left(3 \lambda^{2}-3 \lambda-1\right)\left(\left(X_{1}^{\prime} X_{2}^{\prime} X_{3}\right)_{+}-2\left(X_{1}^{\prime \prime} X_{2} X_{3}\right)_{+}\right) \xi .
\end{aligned}
$$


4) Direct calculation from (2.5) gives the projectively equivariant symbol of a fourth order expression $\left[L_{X_{1}}^{\lambda} L_{X_{2}}^{\lambda} L_{X_{3}}^{\lambda} L_{X_{4}}^{\lambda}\right]_{+}$, that is:

$$
\begin{aligned}
\sigma_{\lambda}\left(\left[L_{X_{1}}^{\lambda} L_{X_{2}}^{\lambda} L_{X_{3}}^{\lambda} L_{X_{4}}^{\lambda}\right]_{+}\right)=\left(X_{1} X_{2} X_{3} X_{4}\right)_{+} \xi^{4} \\
\quad+\frac{1}{7}\left(6 \lambda^{2}-6 \lambda-5\right)\left(\left(X_{1}^{\prime} X_{2}^{\prime} X_{3} X_{4}\right)_{+}-2\left(X_{1}^{\prime \prime} X_{2} X_{3} X_{4}\right)_{+}\right) \xi^{2} \\
\quad+\frac{1}{15} \lambda(\lambda-1)\left(3 \lambda^{2}-3 \lambda-1\right)\left(\left(X_{1}^{\prime} X_{2}^{\prime} X_{3}^{\prime} X_{4}^{\prime}\right)_{+}-4\left(X_{1}^{\prime \prime} X_{2}^{\prime} X_{3}^{\prime} X_{4}\right)_{+}\right. \\
\left.\quad+4\left(X_{1}^{\prime \prime} X_{2}^{\prime \prime} X_{3} X_{4}\right)_{+}\right) .
\end{aligned}
$$

5) In the same manner, one can easily check that the $\mathrm{sl}_{2}$-equivariant symbol of a fifth order expression $\left[L_{X_{1}}^{\lambda} L_{X_{2}}^{\lambda} L_{X_{3}}^{\lambda} L_{X_{4}}^{\lambda} L_{X_{4}}^{\lambda}\right]_{+}$is:

$$
\begin{aligned}
\sigma_{\lambda}([ & \left.\left.L_{X_{1}}^{\lambda} L_{X_{2}}^{\lambda} L_{X_{3}}^{\lambda} L_{X_{4}}^{\lambda} L_{X_{5}}^{\lambda}\right]_{+}\right)=\left(X_{1} X_{2} X_{3} X_{4} X_{5}\right)_{+} \xi^{5} \\
& +\frac{5}{9}\left(2 \lambda^{2}-2 \lambda-3\right)\left(\left(X_{1}^{\prime} X_{2}^{\prime} X_{3} X_{4} X_{5}\right)_{+}-2\left(X_{1}^{\prime \prime} X_{2} X_{3} X_{4} X_{5}\right)_{+}\right) \xi^{3} \\
& +\frac{1}{7}\left(3 \lambda^{4}-6 \lambda^{3}+3 \lambda+1\right)\left(\left(X_{1}^{\prime} X_{2}^{\prime} X_{3}^{\prime} X_{4}^{\prime} X_{5}\right)_{+}-4\left(X_{1}^{\prime \prime} X_{2}^{\prime} X_{3}^{\prime} X_{4} X_{5}\right)_{+}\right. \\
& \left.+4\left(X_{1}^{\prime \prime} X_{2}^{\prime \prime} X_{3} X_{4} X_{5}\right)_{+}\right) \xi
\end{aligned}
$$

\section{Acknowledgments}

I would like to thank V Ovsienko for statement of the problem. I am also grateful to Ch Duval and A El Gradechi for enlightening discussions.

\section{References}

[1] Beilinson A and Bernstein J, Localisation de g-modules, C.R. Acad. Sci. Paris Ser. I Math. 292 (1981), 15-18.

[2] Beilinson A and Bernstein J, A Proof of Jantzen Conjectures, Adv. in Sov. Math. 16 (1993), $1-50$.

[3] Cartan E, Leçons sur la théorie des espaces à connexion projective, Gauthier - Villars, Paris, 1937.

[4] Cohen P, Manin Yu and Zagier D, Automorphic Pseudodifferential Operators, in Progr. Nonlinear Diff. Eq. Appl., Vol. 26, Birkhäuser, Boston, 1997, 17-47.

[5] Duval C and Ovsienko V, Space of Second Order Linear Differential Operators as a Module Over the Lie Algebra of Vector Fields, Adv. in Math. 132, Nr. 2 (1997), 316-333.

[6] Dixmier J, Algèbres enveloppantes, Gauthier - Villars, Paris, 1974.

[7] Feigin B L, The Lie Algebras gl $(\lambda)$ and Cohomologies of Lie Algebra of Differential Operators, Russian Math. Surveys, 43, Nr. 2 (1988), 157-158.

[8] Gargoubi H, Sur la géométrie de l'espace des opérateurs différentiels linéaires sur R, Bull. Soc. Roy. Sci. Liège 69, Nr. 1 (2000), 21-47. 
[9] Gargoubi H and Ovsienko V, Space of Linear Differential Operators on the Real Line as a Module Over the Lie Algebra of Vector Fields, Internat. Mathem. Res. Notices Nr. 5 (1996), $235-251$.

[10] Gargoubi H and Ovsienko V, Modules of Differential Operators on the Real Line, Funct. Anal. Appl. 35, Nr. 1 (2001), 16-22.

[11] Graham R, Knuth D and Patashnik O, Concrete Mathematics, Addison-Wesley, 1989.

[12] Grozman P and Leites D A, Lie Superalgebras of Supermatrices of Complex Size. Their Generalizations and Related Integrable Systems, in Proc. Internatnl. Symp. Complex Analysis and Related Topics, Editors: E. Ramirez de Arellano, et. al., Mexico, 1996, Birkhäuser Verlag, 1999, 73-105.

[13] Khesin B and Malikov F, Universal Drinfeld-Sokolov Reduction and the Lie Algebras of Matrices of Complex Size, Comm. Math. Phys. 175, Nr. 1 (1996), 113-134.

[14] Lecomte P B A, Mathonet P and Tousset E, Comparison of Some Modules of the Lie Algebra of Vector Fields, Indag. Math., N.S. 7, Nr. 4 (1996), 461-471.

[15] Lecomte P B A and Ovsienko V, Projectively Invariant Symbol Calculus, Lett. Math. Phys. 49, Nr. 3 (1999), 173-196.

[16] Leites D A and Sergeev A N, Orthogonal Polynomials of a Discrete Variable and Lie Algebras of Complex-Size Matrices, Theor. Math. Phys. 123, Nr. 2 (2000), 582-608.

[17] Shoikhet B, Certain Topics on the Representation Theory of the Lie Algebra gl $(\lambda)$. Complex Analysis and Representation Theory. 1, J. Math. Sci. (New York) 92, Nr. 2 (1998), 3764-3806.

[18] Wilczynski E J, Projective Differential Geometry of Curves and Ruled Surfaces, Leipzig Teubner, 1906. 